\title{
Dynamic Changes in Serum Markers and Their Utility in the Early Diagnosis of All Stages of Hepatitis B-Associated Hepatocellular Carcinoma
}

This article was published in the following Dove Press journal: OncoTargets and Therapy

\author{
Min $\mathrm{Wu}^{\mathrm{I}}$ \\ Zhaobo Liu' \\ Xin $\mathrm{Li}^{1}$ \\ Aiying Zhang ${ }^{2}$ \\ Ning $\mathrm{Li}^{1,2}$ \\ 'Department of General Surgery, Beijing \\ Youan Hospital, Capital Medical \\ University, Beijing, People's Republic of \\ China; ${ }^{2}$ Beijing Institute of Hepatology, \\ Beijing Youan Hospital, Capital Medical \\ University, Beijing, People's Republic of \\ China
}

\begin{abstract}
Objective: This study aimed to evaluate the individual and combined diagnostic values of serum alpha-fetoprotein (AFP), des-gamma-carboxyprothrombin (DCP), glypican-3 (GPC3) and golgi protein 73 (GP73) in diagnosing hepatitis B virus (HBV)-related hepatocellular carcinoma (HCC).
\end{abstract}

Methods: Participants from Beijing YouAn Hospital were enrolled and divided into seven groups. Serum was collected and the levels of AFP, GPC3, GP73 and DCP were simultaneously measured with a protein array. Pearson's $\chi^{2}$ test was applied to compare the clinicopathological characteristics. Receiver operating characteristic (ROC) curves were used to analyse the diagnostic performance of the four markers.

Results: As a single biomarker for differentiating HCC from all controls, AFP had a larger area under the curve (AUC) $(0.798,95 \%$ CI $(0.754-0.838)$ than the other biomarkers, with a sensitivity of $77.3 \%$ and a specificity of $71.1 \%$. Among the other combinations, AFP plus GPC3 and DCP $(0.871,95 \%$ CI $(0.833-0.903))$ was the best at differentiating HCC from all controls. In discriminating very early stage and early stage HCC from all controls, the AUC of GPC3 (0.744, 95\% CI (0.690-0.793); sensitivity 62.8\%; specificity $83.3 \%)$ was better than that of AFP (0.723, 95\% CI (0.668-0.774); sensitivity 67.3\%; specificity $71.7 \%$ ). Among all biomarker combinations, the combination of AFP, GPC3 and GP73 had the largest AUC (0.843, 95\% CI (0.796-0.883); sensitivity 84.1\%; specificity 71.7\%). AFP (AUC 0.726, 95\% $\mathrm{CI}(0.662-0.784))$ showed the best performance in the very early diagnosis of HBV-related HCC. Conclusion: As a single biomarker, AFP has an advantage in the very early and early diagnosis of HBV-related HCC. The combination of AFP, GPC3 and GP73 is the most suitable marker for the early diagnosis of HBV-related HCC. However, AFP remains the best biomarker for the very early diagnosis of HBV-related HCC, and the adding of one or more markers does not significantly improve the diagnostic accuracy.

Keywords: hepatocellular carcinoma, hepatitis B virus, diagnosis, protein array

\section{Introduction}

Liver cancer is estimated to be the second most common cancer in China, resulting in approximately 38 thousand deaths each year. ${ }^{1,2}$ In particular, the incidence and mortality rates of liver cancer in China are approximately twice as high in males as in females. ${ }^{3}$ Hepatocellular carcinoma (HCC) is the most frequent subtype of liver cancer, accounting for $80 \%$ of liver cancer cases. ${ }^{4}$

Although various risk factors, such as aflatoxin B1 exposure, tobacco smoking, alcohol abuse and hepatitis virus infection, are identified to be closely associated 
with the development of $\mathrm{HCC},{ }^{5}$ hepatitis B virus (HBV) infection remains the major contributor to hepatocarcinogenesis in China. ${ }^{6}$ Chronic HBV infection can lead to the development of liver cirrhosis (LC), which can subsequently transform to $\mathrm{HCC}^{7}$ Moreover, the long-term survival of patients with HCC is largely dependent on the clinical stage at diagnosis. ${ }^{8}$ Thus, the most critical approach for the early diagnosis of HCC is to distinguish malignant nodules from benign nodules in LC.

Ultrasonography is the preferred screening test, and dynamic magnetic resonance imaging and computed tomography are top choices for confirmation tests in the early diagnosis of HCC. ${ }^{9,10}$ However, the per-lesion sensitivity of magnetic resonance imaging and computed tomography for nodular HCCs sized $1-2 \mathrm{~cm}$ is reported to be $44-47 \%$ and 40-44\%, respectively. ${ }^{11}$ Thus, tumour markers, such as alpha-fetoprotein (AFP), ${ }^{12,13}$ des-gamma-

carboxyprothrombin (DCP) ${ }^{13}$ glypican-3 (GPC3) ${ }^{14}$ and golgi protein 73 (GP73), ${ }^{15}$ may be used as complementary modalities in the surveillance and diagnosis of HCC. Because increased AFP levels are observed in LC and active hepatitis, ${ }^{16}$ AFP has been the most frequently used tumour biomarker in the diagnosis of HCC since the 1970s. Liebman et al reported that DCP, an abnormal form of a coagulation protein, was increased in $\mathrm{HCC}$ and was a highly specific marker of HCC. ${ }^{17}$ DCP was estimated to be a better diagnostic marker than AFP for the early diagnosis of HCC. The mRNA levels of GPC3, a heparin sulfate proteoglycan, were found to be increased in $\mathrm{HCC}{ }^{18}$ Although GP73, a resident Golgi-specific membrane protein, was found to be increased gradually in chronic liver disease, it was reported to be the most sensitive biomarker for the diagnosis of early HCC. ${ }^{19}$

The combination of several tumour markers may improve the sensitivity without lowering the specificity. Thus, this study aimed to evaluate the performance of the combination of AFP, GPC3, GP73 and DCP in the early diagnosis of HCC in the context of CHB.

\section{Methods}

\section{Study Population}

A total of 374 subjects from Beijing YouAn Hospital were included in this study and divided into seven groups: the healthy control(HC), CHB, LC, very early stage HCC, early stage $\mathrm{HCC}$, advanced stage $\mathrm{HCC}$ and late stage $\mathrm{HCC}$ groups. The diagnosis of HCC was confirmed by histological examination. The classification of HBV-related HCC was based on the following principles and the Barcelona Clinic Liver Cancer staging classification (2012 version): very early stage $\mathrm{HCC}$ : single nodule $<2 \mathrm{~cm}$; early stage HCC: one to three nodules $<3 \mathrm{~cm}$; advanced stage HCC: multiple nodules; and late stage HCC: vascular invasion or extrahepatic spread. The HCC-related exclusion criteria were combined hepatocellular and cholangiocarcinoma, intrahepatic cholangiocarcinoma, mixed HCC, HCC without HBV infection and HCC with HCV infection. Patients with LC were mainly diagnosed by physical and ultrasound examinations. LC caused by HCV, alcoholic liver disease or cholestasis were excluded from this study. Patients in the LC and CHB groups underwent magnetic resonance imaging and were followed up for six months to exclude potential HCC. Each person gave $5 \mathrm{~mL}$ of serum; blood samples were taken from patients with HCC before they underwent surgery. The serum samples were sub-packed, coded and stored at $-80^{\circ} \mathrm{C}$. This study was approved by the institutional ethics committee of Beijing YouAn Hospital and the written informed consent was obtained from each subject in accordance with the Declaration of Helsinki.

\section{Antibodies, Reagents and Protein Microarray Procedures}

The following antibodies were spotted on aldehyde-coated microscope slides (Shanghai Baiao, China) by a GeSiM NanoPlotter $^{\mathrm{TM}}$ Micropipetting System (Radeberg, Germany): mouse monoclonal AFP antibody $(1.25 \mathrm{mg} / \mathrm{mL}$, Frpon Botech Inc, Shenzhen, China), mouse monoclonal GPC3 antibody ( $1 \mathrm{mg} / \mathrm{mL}$, R\&D Bio-techne, Minneapolis, USA), mouse monoclonal Golgi membrane protein 1 (GOLM1) antibody $(0.5 \mathrm{mg} / \mathrm{mL}$, Novus, Centennial, USA) and mouse monoclonal prothrombin factor II antibody $(2.5 \mathrm{mg} / \mathrm{mL}$, Fujirebio Inc, Tokyo, Japan). Bovine serum albumin (BSA, Abcam, Cambridge, USA) was also spotted as a control. All above reagents were spotted in triplicate at $8^{\circ} \mathrm{C}$ with a $50 \%$ relative humidity. Horseradish peroxidase (HRP) conjugation kits and normal goat serum were purchased from Abcam (Cambridge, USA).

Chemiluminescent HRP substrate was purchased from Merck KgaA Ltd, Darmstadt, Germany. Rabbit HRPconjugated polyclonal AFP antibody $(1 \mu \mathrm{g} / \mathrm{mL}$, Frpon Botech Inc, Shenzhen, China), HRP-conjugated prothrombin factor II antibody $(20 \mu \mathrm{g} / \mathrm{mL}$, Fitzgerald, New York, USA), HRP-conjugated GPC3 antibody $(20 \mu \mathrm{g} / \mathrm{mL}$, R\&D Bio-techne, Minneapolis, USA), and HRP-conjugated polyclonal GOLM1 antibody $(20 \mu \mathrm{g} / \mathrm{mL}$, Novus, Centennial, 
USA) were applied as detection antibodies. The imprinted slides were incubated and blocked by coupling buffer $(10 \%$ normal goat serum with $0.1 \%$ sodium azide) at $37^{\circ} \mathrm{C}$ for 90 mins, rinsed with 1 X PBS supplemented with $1 \%$ Tween-20(PBST0.1) three times for three minutes each, and stored at $4^{\circ} \mathrm{C}$ until use. Then, $15 \mu \mathrm{L}$ of each serum sample was added to the slides, which were then incubated at $37^{\circ} \mathrm{C}$ for 30 mins. Fifteen microliters of a detection antibody mixture were added to each microscope after it was washed three times for three minutes each with PBST0.1, and the slides were incubated at $37^{\circ} \mathrm{C}$ for 30 mins. The slides were finally scanned using Chemi Doc ${ }^{\mathrm{TM}}$ MP System (Bio-Rad, California, USA) after adding the chemiluminescent HRP substrate. Image Lab was used to quantify the data. The above protein microarray technology was built and validated previously in our laboratory and was patents were applied for in China (ZL201410069738.3) and Australia (201610515896.6). All the samples were measured at least three times to reduce deviation. Moreover, the investigators processing the biomarker assays were blinded to the clinical sample data.

\section{Statistical Analysis}

The data were analysed in GraphPad Software version 7 (GraphPad Software, San Diego, California) and SPSS version 22.0 for Windows (IBM, Armonk, New York, USA). The clinicopathological characteristics were compared with Pearson's $\chi^{2}$ test. Significant differences were identified with Student's $t$ test for normally distributed data and the Mann-Whitney test for non-normally distributed data. A binary logistic regression model was built to evaluate the calibration power of the biomarkers for HCC diagnosis. The sensitivity, specificity and accuracy were calculated using ROC analysis. The best cut-off value was selected based on the largest value of the Youden index. The criterion for statistical significances was $P<0.05$.

\section{Results}

\section{Baseline Participants Characteristics and Serum AFP, GPC3, GP73 and DCP Levels}

A total of 374 persons were recruited for this study and classified into seven subgroups. The clinicopathological features of the $\mathrm{HC}, \mathrm{CHB}, \mathrm{LC}$ and $\mathrm{HBV}$-related $\mathrm{HCC}$ groups are presented in Table 1 . The seven subgroups were well matched in age $(P=0.08)$, and there was a male predominance in most groups $(P=0.03)$. There were significant differences among the seven subgroups in total bilirubin (TBil), alanine aminotransferase (ALT), aspartate aminotransferase (AST) and total protein (TP) (all $P<0.0001$ ).

The serum levels of AFP, GPC3, GP73 and DCP in all subgroups are presented in Table 1 and Figure 1. AFP levels were significantly higher in the very early stage $\mathrm{HCC}$ group than in the $\mathrm{HC}, \mathrm{CHB}$ and $\mathrm{LC}$ groups (all $P<0.0001$ ) (Figure 1A). However, AFP levels in the advanced stage and late stage HCC groups, but not the early stage $\mathrm{HCC}$ group, were significantly higher than those in the very early stage HCC group $(P<0.0001)$ (Figure 1A). Compared with the $\mathrm{HC}$ group, the $\mathrm{CHB}$, LC and very early stage HCC groups had significantly higher serum GPC3 levels (Figure 1B). GPC3 levels were significantly higher in the advanced stage and late stage HCC groups than in the very early stage HCC group (Figure 1B). However, there were no significant differences in GPC3 levels between the very early stage HCC group and the $\mathrm{CHB}, \mathrm{LC}$ and advanced stage HCC groups (Figure 1B). For GP73, there were no significant differences among the very early stage HCC, LC, CHB and HC groups, whereas GP73 levels were significantly lower in the very early stage HCC group than in the early, advanced and late stage $\mathrm{HCC}$ groups, indicating that GP73 may not be a good biomarker for the very early diagnosis of HBV-related HCC (Figure 1C). For DCP, there were no significant differences between the LC and very early stage HCC groups (Figure 1D). DCP levels were significantly higher in the late stage HCC group than in the very early, early and advanced stage HCC groups, indicating that an elevated serum DCP level may be a biomarker of poor prognosis in HBV-related HCC (Figure 1D). Typical representative images of the biomarkers in each group are presented in Figure 1E.

\section{The Ability of AFP, GPC3, GP73 and DCP to Differentiate HBV-Related HCC from Controls}

The ROC curve analysis showed that as a single biomarker for differentiating HCC from all controls, AFP had a larger AUC value $(0.798,95 \%$ CI $(0.754-0.838))$ than GPC3, GP73 and DCP, with a sensitivity of $77.3 \%$ and a specificity of $71.1 \%$. AFP plus GPC3, GP73 or DCP had a higher AUC value, sensitivity and specificity than AFP alone in differentiating HCC from all controls (Table 2 and Figure 2A). However, AFP plus two of 
Table I The Clinicopathological Characteristics of the Study Population

\begin{tabular}{|c|c|c|c|c|c|c|c|c|}
\hline Features & $\begin{array}{l}\mathrm{HC} \\
n=50\end{array}$ & $\begin{array}{l}\mathrm{CHB} \\
\mathrm{n}=50\end{array}$ & $\begin{array}{l}\text { LC } \\
n=76\end{array}$ & $\begin{array}{l}\text { Very Stage HCC } \\
n=40\end{array}$ & $\begin{array}{l}\text { Early Stage HCC } \\
n=73\end{array}$ & $\begin{array}{l}\text { Advanced HCC } \\
n=45\end{array}$ & $\begin{array}{l}\text { Late Stage HCC } \\
n=40\end{array}$ & $P$ value \\
\hline \multicolumn{9}{|l|}{ Age, years } \\
\hline$\leq 55$ & 37 & 31 & 46 & 22 & 37 & 23 & 18 & 0.08 \\
\hline$>55$ & 13 & 19 & 30 & 18 & 36 & 22 & 22 & \\
\hline \multicolumn{9}{|l|}{ Gender } \\
\hline Male & 30 & 24 & 44 & 28 & 56 & 37 & 23 & 0.03 \\
\hline Female & 20 & 26 & 32 & 12 & 17 & 8 & 17 & \\
\hline \multicolumn{9}{|l|}{ Child-pugh } \\
\hline A & 50 & 50 & 59 & 35 & 64 & 45 & 32 & $<0.0001$ \\
\hline B & 0 & 0 & 12 & 5 & 8 & 0 & 8 & \\
\hline C & 0 & 0 & 5 & 0 & 1 & 0 & 0 & \\
\hline \multicolumn{9}{|l|}{ TBil, umol/L } \\
\hline$\leq 21$ & 50 & 47 & 46 & 25 & 53 & 37 & 30 & $<0.0001$ \\
\hline$>21$ & 0 & 3 & 30 & 15 & 20 & 8 & 10 & \\
\hline \multicolumn{9}{|l|}{ ALT, U/L } \\
\hline$\leq 50$ & 50 & 50 & 54 & 33 & 54 & 34 & 31 & $<0.0001$ \\
\hline$>50$ & 0 & 0 & 22 & 7 & 19 & 11 & 9 & \\
\hline \multicolumn{9}{|l|}{ AST, U/L } \\
\hline$\leq 40$ & 50 & 40 & 59 & 33 & 53 & 23 & 23 & $<0.0001$ \\
\hline$>40$ & 0 & 10 & 17 & 7 & 20 & 22 & 17 & \\
\hline \multicolumn{9}{|l|}{ TP, g/L } \\
\hline$\leq 65$ & 0 & 2 & 19 & 10 & 26 & 13 & 9 & $<0.0001$ \\
\hline$>65$ & 50 & 48 & 57 & 30 & 47 & 32 & 31 & \\
\hline \multicolumn{9}{|l|}{ Tumor differentiation } \\
\hline Well and moderately & & & & 28 & 48 & 16 & 18 & 0.01 \\
\hline Poorly & & & & 12 & 25 & 29 & 22 & \\
\hline \multicolumn{9}{|l|}{ Cirrhosis } \\
\hline Yes & 0 & 0 & 76 & 25 & 45 & 20 & 18 & $<0.0001$ \\
\hline No & 50 & 50 & 0 & 15 & 28 & 25 & 22 & \\
\hline \multicolumn{9}{|l|}{ Markers, median (range) } \\
\hline AFP (int) & $0(0,0)$ & $0(0,5431)$ & $0(0,983 \mathrm{I})$ & $575(0,23,249)$ & $309.3(0,30,402)$ & $7036(0,53,824)$ & $|2,05|(0,43,395)$ & $<0.0001$ \\
\hline GPC3(int) & $0(0,0)$ & $0(0,2309)$ & $0(0,5800)$ & $0(0,3272)$ & $0(0,6067)$ & $0(0,4807)$ & $0(0,10,988)$ & $<0.0001$ \\
\hline GP73(int) & $0(0,0)$ & $0(0,0)$ & $0(0,4626)$ & $0(0,0)$ & $0(0,1558)$ & $0(0,4657)$ & $0(0,3|8|)$ & $<0.0001$ \\
\hline $\mathrm{DCP}(\mathrm{int})$ & $0(0,0)$ & $0(0,202.7)$ & $0(0,942 I)$ & $0(0,1|8|)$ & $0(0,5424)$ & $0(0,48 \mid 3)$ & $0(0,9535)$ & $<0.0001$ \\
\hline
\end{tabular}

Abbreviations: HCC, hepatocellular carcinoma; LC, liver cirrhosis; CHB, chronic hepatitis B virus infection; HC, healthy controls; TBil, total bilirubin ALT, alanine aminotransferase; AST, aspartate aminotransferase; TP, total protein; AFP, $\alpha$-fetoprotein; GPC3, glypican 3; GP73, golgi protein 73; DCP, des- $\gamma$-carboxy prothrombin.

GPC3, GP73 or DCP had an even higher AUC value, with no increase or even a decrease in sensitivity, than AFP plus GPC3 in differentiating HCC from all controls (Table 2 and Figure 2A). The combination of the four markers showed the same trend in the diagnosis of HCC. AFP plus GPC3 and GP73 (AUC 0.871, 95\% CI (0.$833-0.8903$ ), sensitivity $70.2 \%$, specificity $89.4 \%$ ) was the best combination for differentiating $\mathrm{HCC}$ from all controls; this combination was better than AFP plus GPC3(AUC $0.863,95 \%$ CI (0.824-0.896), sensitivity $86.9 \%$, specificity $71.7 \%$ ) (Table 2 and Figure 2A).
When differentiating $\mathrm{HCC}$ from $\mathrm{LC}$ and $\mathrm{CHB}$, AFP plus GPC3 and GP73 had a larger AUC $(0.841,95 \%$ CI (0.796-0.879), sensitivity $70.2 \%$, specificity $85.4 \%$ ) than AFP alone (AUC $0.765,95 \%$ CI (0.715-0.810), sensitivity 61.6\%, specificity 73.1\%) (Table 2 and Figure 2B). When differentiating HCC from LC, AFP plus GPC3 and GP73 (AUC $0.835,95 \%$ CI (0.785-0.877), sensitivity 70.2\%, specificity $82.7 \%$ ) remained the best diagnostic combination among individual and combined markers, including AFP (AUC 0.775, 95\% CI (0.721-0.824), sensitivity $77.3 \%$, specificity $66.7 \%$ ) (Table 2 and Figure 2C). 
A

\section{AFP}
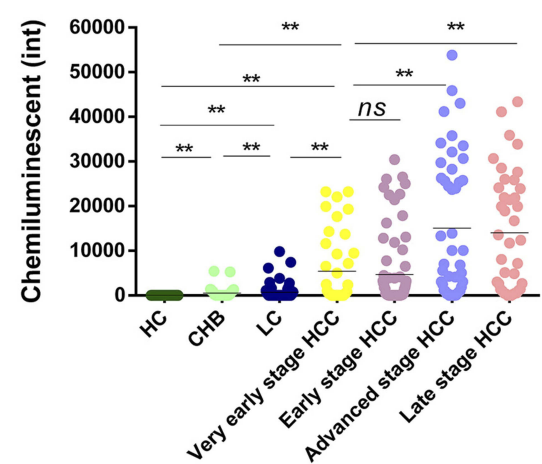

C

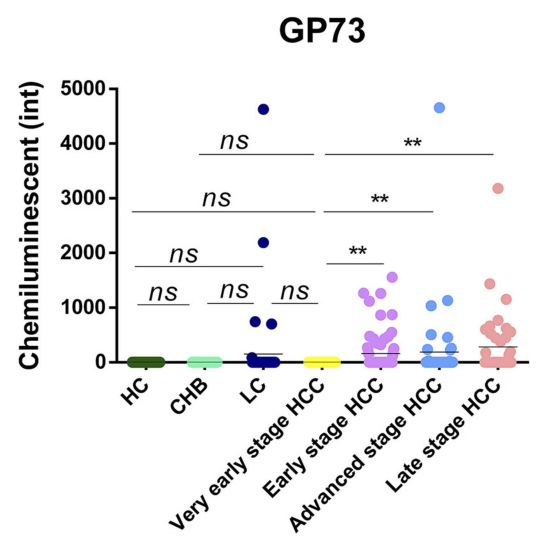

E

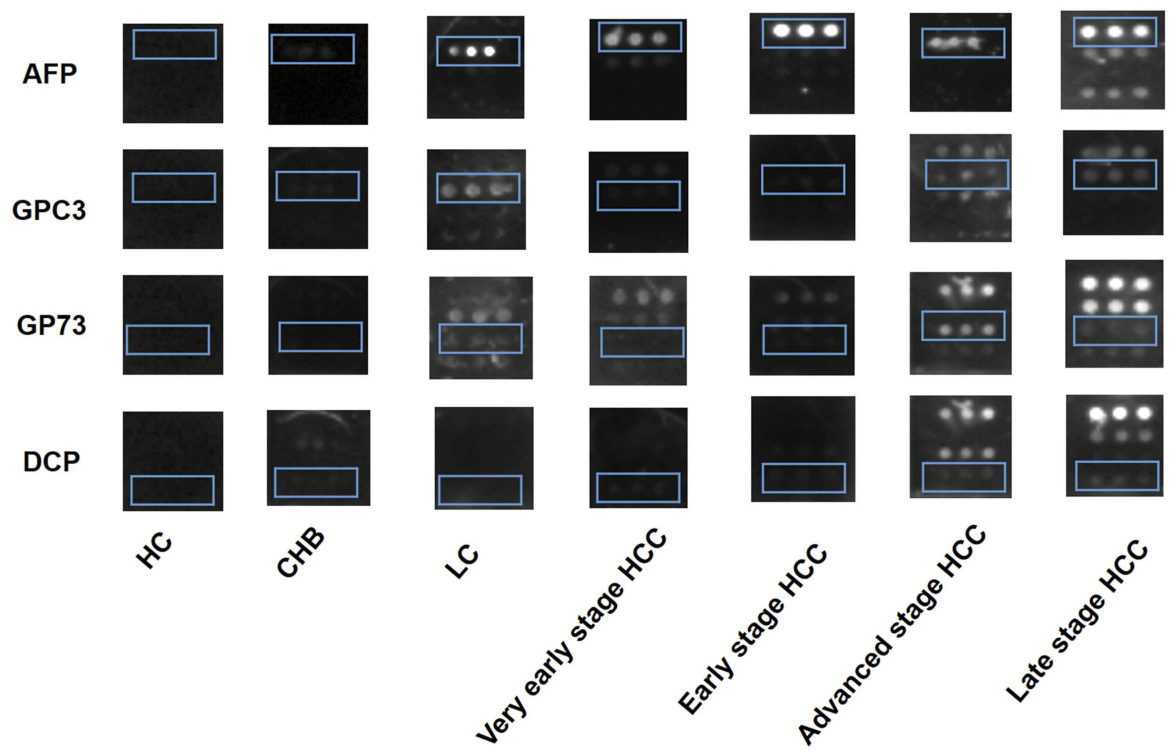

B

GPC3

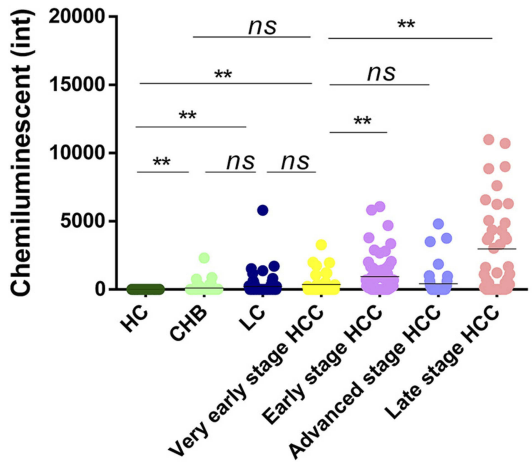

D

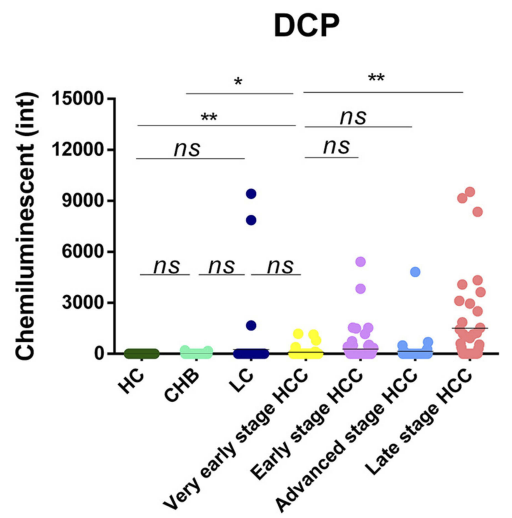

Figure I The levels of serum AFP, GPC3, GP73 and DCP in each subgroup. (A) AFP. (B) GPC3. (C) GP73. (D) DCP. (E) Representative image of each subgroup. *P<0.05, $* * P<0.01$.

Abbreviations: HCC, hepatocellular carcinoma; LC, liver cirrhosis; CHB, chronic hepatitis B virus infection; HC, healthy controls; AFP, $\alpha$-fetoprotein; GPC3, glypican 3; GP73, golgi protein 73; DCP, des- $\gamma$-carboxy prothrombin; ns, no significance; int, intensity. 
Table 2 The Value of Serum AFP, GPC3, GP73 and DCP in the Diagnosis of HCC (Including All HCC Patients)

\begin{tabular}{|c|c|c|c|c|c|c|}
\hline Biomarkers & $\operatorname{AUC}(95 \% \mathrm{Cl})$ & $P$ value & Sen(\%) & Spe (\%) & PPV(\%) & NPV(\%) \\
\hline \multicolumn{7}{|l|}{ vs $\mathrm{LC}, \mathrm{CHB}, \mathrm{HC}$} \\
\hline AFP & $0.798(0.754-0.838)$ & $<0.0001$ & 77.3 & 71.1 & 74.6 & 74.0 \\
\hline GPC3 & $0.73 \mid(0.684-0.775)$ & $<0.0001$ & 58.6 & 84.4 & 80.6 & 65.0 \\
\hline GP73 & $0.616(0.565-0.665)$ & $<0.0001$ & 23.7 & 99.4 & 97.9 & 54.2 \\
\hline $\mathrm{DCP}$ & $0.634(0.583-0.683)$ & $<0.0001$ & 29.8 & 97.2 & 92.2 & 55.7 \\
\hline $\mathrm{AFP}+\mathrm{GPC} 3$ & $0.863(0.824-0.896)$ & $<0.0001$ & 86.9 & 71.7 & 77.1 & 83.2 \\
\hline $\mathrm{AFP}+\mathrm{GP73}$ & $0.83 \mathrm{I}(0.790-0.868)$ & $<0.0001$ & 60.1 & 95.6 & 93.7 & 68.5 \\
\hline $\mathrm{AFP}+\mathrm{DCP}$ & $0.810(0.767-0.848)$ & $<0.0001$ & 80.8 & 70.6 & 75.1 & 77.0 \\
\hline GPC3+GP73 & $0.758(0.7 \mid 2-0.80 I)$ & $<0.0001$ & 62.6 & 84.4 & 81.6 & 67.3 \\
\hline GPC3+DCP & $0.753(0.706-0.796)$ & $<0.0001$ & 64.1 & 83.3 & 80.9 & 67.9 \\
\hline GP73+DCP & $0.684(0.635-0.731)$ & $<0.0001$ & 39.4 & 97.2 & 94.0 & 59.3 \\
\hline $\mathrm{AFP}+\mathrm{GPC} 3+\mathrm{GP73}$ & $0.87 I(0.833-0.903)$ & $<0.0001$ & 70.2 & 89.4 & 88.0 & 73.2 \\
\hline $\mathrm{AFP}+\mathrm{GPC} 3+\mathrm{DCP}$ & $0.863(0.824-0.896)$ & $<0.0001$ & 86.9 & 71.7 & 77.1 & 83.2 \\
\hline GPC3+GP73+DCP & $0.77 \mid(0.725-0.8 \mid 2)$ & $<0.0001$ & 66.2 & 83.3 & 81.4 & 69.1 \\
\hline $\mathrm{AFP}+\mathrm{GPC} 3+\mathrm{GP73}+\mathrm{DCP}$ & $0.867(0.829-0.900)$ & $<0.0001$ & 69.7 & 89.4 & 87.9 & 72.9 \\
\hline \multicolumn{7}{|l|}{ vs LC,CHB } \\
\hline AFP & $0.765(0.715-0.810)$ & $<0.0001$ & 61.6 & 73.1 & 77.7 & 55.6 \\
\hline GPC3 & $0.706(0.653-0.754)$ & $<0.0001$ & 58.6 & 78.5 & 80.6 & 55.4 \\
\hline GP73 & $0.614(0.559-0.667)$ & $<0.0001$ & 23.7 & 99.2 & 97.9 & 46.1 \\
\hline $\mathrm{DCP}$ & $0.628(0.574-0.68 I)$ & $<0.0001$ & 29.8 & 96.2 & 92.2 & 47.3 \\
\hline $\mathrm{AFP}+\mathrm{GPC} 3$ & $0.830(0.785-0.869)$ & $<0.0001$ & 63.6 & 87.7 & 88.7 & 61.3 \\
\hline $\mathrm{AFP}+\mathrm{GP73}$ & $0.802(0.755-0.844)$ & $<0.0001$ & 60.1 & 93.8 & 93.7 & 60.7 \\
\hline AFP+DCP & $0.774(0.725-0.818)$ & $<0.0001$ & 51.5 & 93.8 & 92.7 & 56.0 \\
\hline GPC3+GP73 & $0.735(0.684-0.782)$ & $<0.0001$ & 62.6 & 78.5 & 81.6 & 58.0 \\
\hline GPC3+DCP & $0.725(0.673-0.773)$ & $<0.0001$ & 64.1 & 76.9 & 80.9 & 58.5 \\
\hline GP73+DCP & $0.679(0.626-0.729)$ & $<0.0001$ & 39.4 & 96.2 & 94.0 & 51.0 \\
\hline AFP+GPC3+GP73 & $0.84 I(0.796-0.879)$ & $<0.0001$ & 70.2 & 85.4 & 88.0 & 65.3 \\
\hline $\mathrm{AFP}+\mathrm{GPC} 3+\mathrm{DCP}$ & $0.830(0.785-0.869)$ & $<0.0001$ & 63.6 & 87.7 & 88.7 & 61.3 \\
\hline GPC3+GP73+DCP & $0.746(0.695-0.792)$ & $<0.0001$ & 66.2 & 76.9 & 81.4 & 59.9 \\
\hline $\mathrm{AFP}+\mathrm{GPC} 3+\mathrm{GP73}+\mathrm{DCP}$ & $0.837(0.793-0.876)$ & $<0.0001$ & 64.1 & 91.5 & 92.0 & 62.6 \\
\hline \multicolumn{7}{|l|}{ vs LC } \\
\hline AFP & $0.775(0.72 \mathrm{I}-0.824)$ & $<0.0001$ & 77.3 & 66.7 & 86.0 & 52.6 \\
\hline GPC3 & $0.696(0.638-0.750)$ & $<0.0001$ & 58.6 & 77.3 & 87.2 & $4 I .4$ \\
\hline GP73 & $0.6 \mathrm{II}(0.55 \mathrm{I}-0.670)$ & $<0.0001$ & 23.7 & 98.7 & 97.9 & 32.9 \\
\hline $\mathrm{DCP}$ & $0.624(0.564-0.682)$ & $<0.0001$ & 29.8 & 96.0 & 95.2 & 34.1 \\
\hline $\mathrm{AFP}+\mathrm{GPC} 3$ & $0.824(0.774-0.868)$ & $<0.0001$ & 68.2 & 81.3 & 90.1 & 48.8 \\
\hline AFP+GP73 & $0.810(0.758-0.855)$ & $<0.0001$ & 60.1 & 92.0 & 95.2 & 46.6 \\
\hline $\mathrm{AFP}+\mathrm{DCP}$ & $0.784(0.730-0.831)$ & $<0.0001$ & 80.8 & 65.3 & 86.0 & 56.3 \\
\hline GPC3+GP73 & $0.727(0.670-0.779)$ & $<0.0001$ & 62.6 & 77.3 & 87.9 & 43.9 \\
\hline GPC3+DCP & $0.709(0.65 I-0.762)$ & $<0.0001$ & 64.1 & 74.7 & 87.0 & 44.1 \\
\hline GP73+DCP & $0.675(0.616-0.730)$ & $<0.0001$ & 39.4 & 96.0 & 96.3 & 37.5 \\
\hline $\mathrm{AFP}+\mathrm{GPC} 3+\mathrm{GP73}$ & $0.835(0.785-0.877)$ & $<0.0001$ & 70.2 & 82.7 & 91.4 & 51.2 \\
\hline$A F P+G P C 3+D C P$ & $0.825(0.774-0.868)$ & $<0.0001$ & 66.7 & 82.7 & 91.0 & 48.4 \\
\hline GPC3+GP73+DCP & $0.73 \mid(0.674-0.783)$ & $<0.0001$ & 66.2 & 74.7 & 87.3 & 45.5 \\
\hline $\mathrm{AFP}+\mathrm{GPC} 3+\mathrm{GP73}+\mathrm{DCP}$ & $0.833(0.783-0.875)$ & $<0.0001$ & 64.1 & 89.3 & 94.1 & 48.6 \\
\hline
\end{tabular}

Abbreviations: AFP, $\alpha$-fetoprotein; GPC3, glypican 3; GP73, golgi protein 73; DCP, des- $\gamma$-carboxy prothrombin; AUC, area under curve; Sen, sensitivity; Sep, specificity; PPV, positive predictive value; NPV, negative predictive value; HCC, hepatocellular carcinoma; LC, liver cirrhosis; $\mathrm{CHB}$, chronic hepatitis B virus infection; HC, healthy controls. 

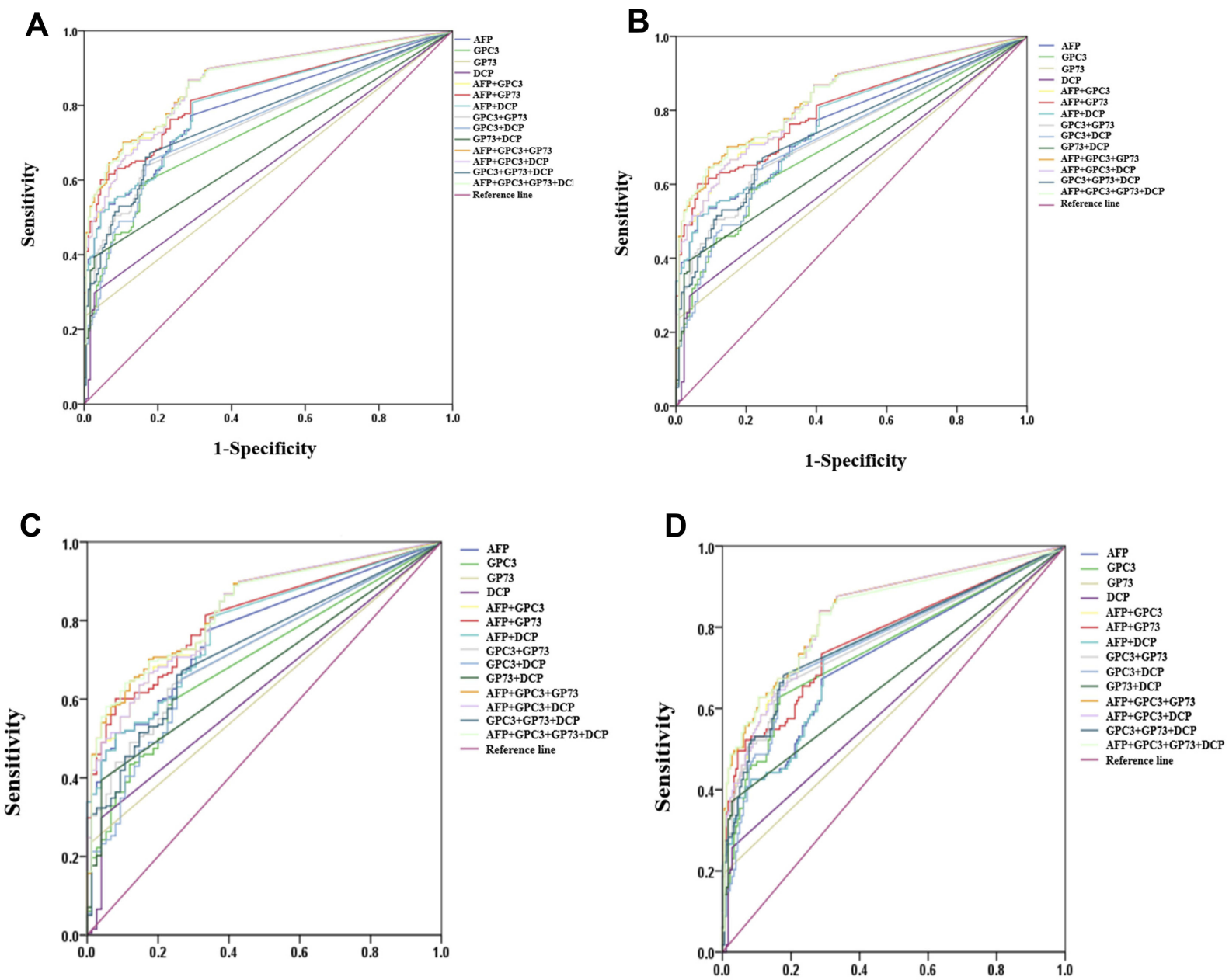

1-Specificity

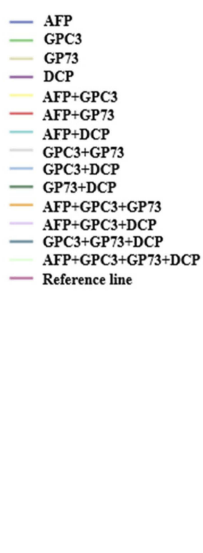

1-Specificity

E
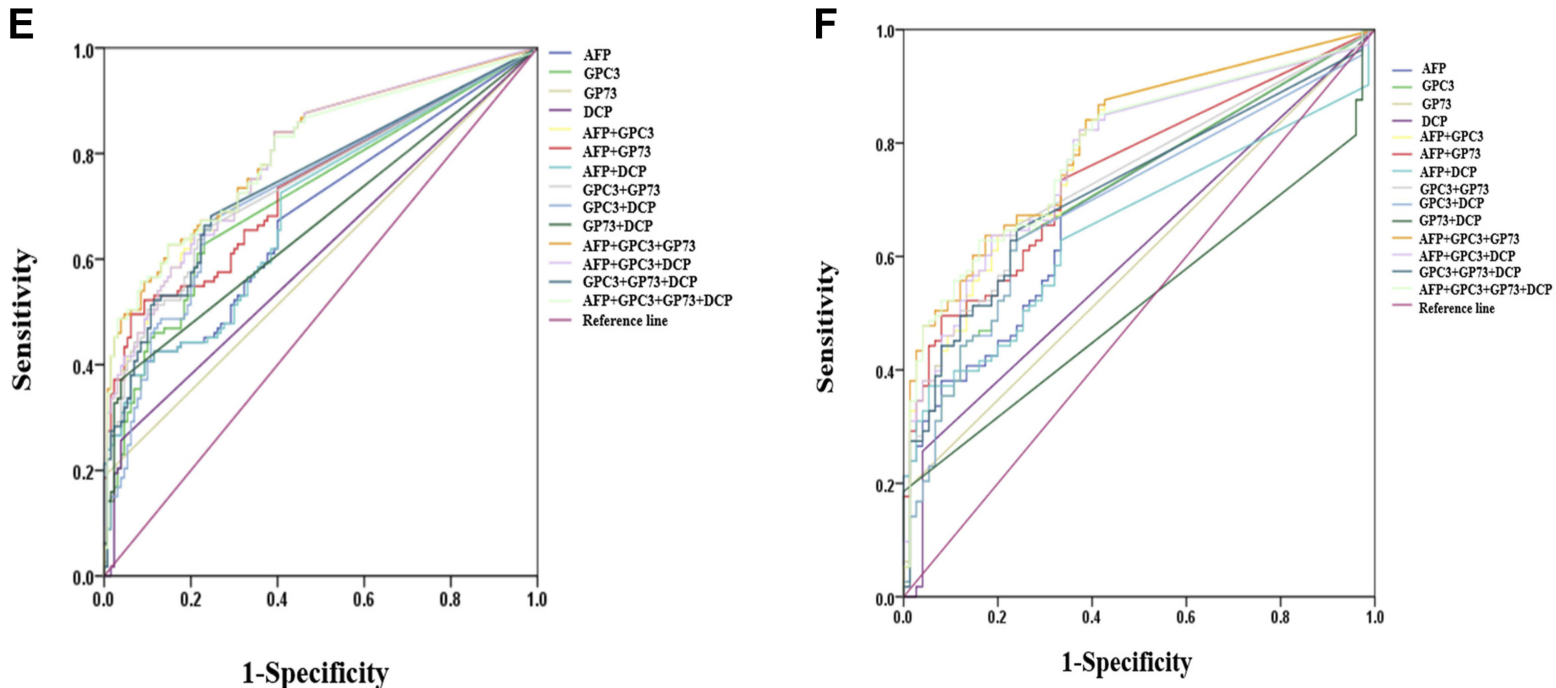

1-Specificity

Figure 2 Assessment of the diagnostic value of serum AFP, GPC3, GP73 and DCP in differentiating HBV-related HCC from controls. (A) All HCC vs LC, CHB, HC. (B) All HCC vs LC, CHB. (C) All HCC vs LC. (D) Very early and early stage HCC vs LC, CHB, HC. (E) Very early and early stage HCC vs LC, CHB. (F) Very early and early stage HCC vs LC.

Abbreviations: HCC, hepatocellular carcinoma; LC, liver cirrhosis; CHB, chronic hepatitis B virus infection; HC, healthy controls; AFP, $\alpha$-fetoprotein; GPC3, glypican 3; GP73, golgi protein 73; DCP, des- $\gamma$-carboxy prothrombin. 
The Ability of AFP, GPC3, GP73 and DCP to Differentiate Very Early and Early Stage HBV-Related HCC from Controls

In discriminating very early and early stage $\mathrm{HCC}$ from all controls, the AUC values of AFP and GPC3 were 0.723 (95\% CI (0.668-0.774), sensitivity 67.3\%, specificity $71.7 \%$ ), 0.744 (95\% CI (0.690-0.793), sensitivity 62.8\%, specificity $83.3 \%$ ), respectively (Table 3 and Figure 2D). The combination of AFP and GPC3 had a better AUC (0.833, 95\% CI (0.786-0.874), sensitivity 84.1\%, specificity 71.7\%) than AFP, GPC3, AFP plus GP73 (AUC $0.773,95 \%$ CI $(0.721-0.820)$ ) or AFP plus DCP (AUC $0.740,95 \%$ CI (0.686-0.790)) (Table 3 and Figure 2D). The combination of AFP, GPC3 and GP73 had a larger AUC (0.843, 95\% CI (0.796-0.883), sensitivity 84.1\%, specificity $71.7 \%$ ) than the other combinations of markers (Table 3 and Figure 2D). The above trends in markers performance were similar to those for distinguishing very early and early stage HCC from LC with or without $\mathrm{CHB}$ (Table 3 and Figure 2E and F).

\section{The Ability of AFP, GPC3, GP73 and DCP to Differentiate Very Early Stage HBV-Related HCC from Controls}

As a single marker, AFP (AUC 0.726, 95\% CI (0.662-0.784), sensitivity $65.0 \%$, specificity $76.7 \%$ ) showed the best performance in differentiating very early stage HCC from all controls. Among the biomarker combinations, AFP plus GPC3 (AUC 0.751, 95\% CI (0.688-0.807), sensitivity 75.0\%, specificity 71.7\%) and AFP plus GPC3 and GP73(AUC 0.752, 95\% CI (0.689-0.807), sensitivity 75.0\%, specificity 71.7\%) had similar diagnostic performance. However, a comparison of the ROC curves showed no significant differences among the AUC values for AFP, AFP plus GPC3, AFP plus GPC3 and GP73, and the four-marker combination $(P>0.05)$ (Table 4 and Figure $3 \mathrm{~A}$ ). When differentiating very early stage HBVrelated $\mathrm{HCC}$ from $\mathrm{LC}$ and $\mathrm{CHB}$, the AUC values of AFP and AFP plus GPC3 were 0.688 (95\% CI (0.613-0.757), sensitivity $42.5 \%$, specificity $96.2 \%)$ and $0.703 \quad(95 \% \quad$ CI (0.629-0.771), sensitivity 42.5\%, specificity 96.2\%); those markers showed the best diagnostic performance. However, all biomarker combinations had the same low sensitivity (42.5\%). Moreover, a comparisons of the ROC curves showed that the AUC values of AFP plus GCP3 and of AFP, GPC3 and GP73 or DCP were not significantly higher than that of AFP $(P>0.05)$ (Table 4 and Figure 3B). When differentiating very early stage HBV-related HCC from LC, the AUC of AFP was $0.708(95 \% \mathrm{CI}(0.616-0.789))$, with a sensitivity of $75.0 \%$ and a specificity of $62.7 \%$, and the addition of one or more markers did not improve the diagnostic accuracy (Table 4 and Figure 3C).

\section{Discussion}

In the current study, we investigated the role of four common liver cancer serum markers (AFP, DCP, GPC3 and GP73) in the diagnosis of liver cancer and other benign liver diseases caused by HBV. We found that when a single marker was used to diagnose HBV-related HCC in general or at an early stage, AFP was a relatively and effective discriminator compared to the other three biomarkers, with good sensitivity and specificity. Regarding the associated diagnosis, AFP combined with GPC3 and GP73 had the largest AUC value in differentiating HBV-related $\mathrm{HCC}$ from controls with or without LC, and continuing to increase the number of markers to four, did not increase, or even decreased the overall diagnostic efficiency. The strict control of the temperature and humidity for chip preparation and incubation, appropriate concentrations of antibodies, appropriate rinse temperature and rinse time are required for the accuracy of measurement results. (To reviewer 1)

In contrast to previous studies, the study involved a detailed subgroup analysis of the enrolled population. Non-HCC controls included HCs, patients with $\mathrm{CHB}$ and LC caused by HBV. This study provides a good reference for the diagnosis and identification of a specific types of HBV-related HCC over the entire disease course. The serum levels of these four serum markers showed significant changes in different subpopulations and the overall levels showed similar trends as those in previous studies. That is, GP73 was significantly increased in only patients with $\mathrm{HCC}$, while the other three markers were at certain levels in patients with CHB and HBV-LC. However, all four markers were significantly elevated in patients with late stage HCC. Therefore, we selected these four markers for subsequent diagnostic analysis. Interestingly, in the non$\mathrm{HCC}$ group, which included HCs and patients with $\mathrm{CHB}$, the overall results were consistent regardless of biomarker combination. In the early diagnosis of HBV-related HCC, AFP remained the best individual marker and the combination of AFP, GPC3 and GP73 had the highest diagnostic value; the addition of more markers did not increase; and some cases even reduced, the sensitivity and specificity. Moreover, AFP remained the most suitable marker for the very early diagnosis of $\mathrm{HBV}$-related $\mathrm{HCC}$. 
Table 3 The Value of Serum AFP, GPC3, GP73 and DCP in the Early Diagnosis of HCC (Including the Very Early HCC)

\begin{tabular}{|c|c|c|c|c|c|c|}
\hline Biomarkers & AUC $(95 \% \mathrm{Cl})$ & $P$ value & Sen (\%) & Spe (\%) & PPV (\%) & NPV (\%) \\
\hline \multicolumn{7}{|l|}{ vs LC, CHB, HC } \\
\hline AFP & $0.723(0.668-0.774)$ & $<0.0001$ & 67.3 & 71.7 & 59.4 & 77.6 \\
\hline GPC3 & $0.744(0.690-0.793)$ & $<0.0001$ & 62.8 & 83.3 & 70.3 & 78.1 \\
\hline DCP & $0.613(0.555-0.669)$ & $<0.0001$ & 25.7 & 97.2 & 85.3 & 67.6 \\
\hline $\mathrm{AFP}+\mathrm{GPC} 3$ & $0.833(0.786-0.874)$ & $<0.0001$ & 84.1 & 71.7 & 65.1 & 87.7 \\
\hline AFP+GP73 & $0.773(0.721-0.820)$ & $<0.0001$ & 52.2 & 93.3 & 83.1 & 75.7 \\
\hline AFP+DCP & $0.740(0.686-0.790)$ & $<0.0001$ & 72.6 & 70.6 & 60.7 & 80.4 \\
\hline GPC3+GP73 & $0.766(0.713-0.813)$ & $<0.0001$ & 65.5 & 83.3 & 72.0 & 78.8 \\
\hline GPC3+DCP & $0.759(0.705-0.806)$ & $<0.0001$ & 67.3 & 82.2 & 70.4 & 80.0 \\
\hline GP73+DCP & $0.672(0.616-0.726)$ & $<0.0001$ & 37.2 & 97.2 & 89.4 & 71.1 \\
\hline $\mathrm{AFP}+\mathrm{GPC} 3+\mathrm{GP73}$ & $0.843(0.796-0.883)$ & $<0.0001$ & 84.1 & 71.7 & 63.8 & 85.5 \\
\hline$A F P+G P C 3+D C P$ & $0.833(0.785-0.874)$ & $<0.0001$ & 84.1 & 71.7 & 65.1 & 87.8 \\
\hline GPC3+GP73+DCP & $0.773(0.720-0.819)$ & $<0.0001$ & 68.1 & 82.2 & 70.1 & 79.6 \\
\hline $\mathrm{AFP}+\mathrm{GPC} 3+\mathrm{GP73}+\mathrm{DCP}$ & $0.837(0.789-0.877)$ & $<0.0001$ & 83.2 & 71.7 & 64.8 & 87.2 \\
\hline \multicolumn{7}{|l|}{ vs LC, CHB } \\
\hline AFP & $0.680(0.617-0.738)$ & $<0.0001$ & 57.5 & 64.6 & 58.6 & 63.6 \\
\hline GPC3 & $0.717(0.656-0.773)$ & $<0.0001$ & 62.8 & 76.9 & 70.3 & 70.4 \\
\hline $\mathrm{DCP}$ & $0.607(0.543-0.669)$ & $<0.0001$ & 25.7 & 96.2 & 85.3 & 59.8 \\
\hline $\mathrm{AFP}+\mathrm{GPC} 3$ & $0.793(0.737-0.842)$ & $<0.0001$ & 84.1 & 60.8 & 65.1 & 81.4 \\
\hline AFP+GP73 & $0.737(0.677-0.79 \mid)$ & $<0.0001$ & 49.6 & 93.8 & 87.5 & 68.2 \\
\hline$A F P+D C P$ & $0.693(0.631-0.750)$ & $<0.0001$ & 38.1 & 93.8 & 84.3 & 63.5 \\
\hline GPC3+GP73 & $0.742(0.683-0.796)$ & $<0.0001$ & 65.5 & 76.9 & 72.0 & 71.3 \\
\hline GPC3+DCP & $0.729(0.668-0.783)$ & $<0.0001$ & 67.3 & 75.4 & 70.4 & 72.6 \\
\hline GP73+DCP & $0.667(0.604-0.726)$ & $<0.0001$ & 37.2 & 96.2 & 89.4 & 63.8 \\
\hline $\mathrm{AFP}+\mathrm{GPC} 3+\mathrm{GP73}$ & $0.806(0.751-0.854)$ & $<0.0001$ & 62.8 & 85.4 & 78.9 & 72.5 \\
\hline$A F P+G P C 3+D C P$ & $0.739(0.736-0.842)$ & $<0.0001$ & 84.1 & 60.8 & 65.1 & 81.4 \\
\hline GPC3+GP73+DCP & $0.747(0.687-0.800)$ & $<0.0001$ & 68.1 & 75.4 & 71.4 & 72.5 \\
\hline$A F P+G P C 3+G P 73+D C P$ & $0.80 I(0.746-0.850)$ & $<0.0001$ & 62.8 & 85.4 & 78.9 & 72.5 \\
\hline \multicolumn{7}{|l|}{ vs LC } \\
\hline AFP & $0.697(0.625-0.761)$ & $<0.0001$ & 67.26 & 66.67 & 75.2 & 57.5 \\
\hline GPC3 & $0.707(0.636-0.77 \mid)$ & $<0.0001$ & 62.8 & 76.0 & 79.8 & 57.6 \\
\hline $\mathrm{DCP}$ & $0.603(0.530-0.674)$ & $<0.0001$ & 25.7 & 96.0 & 90.6 & 46.2 \\
\hline $\mathrm{AFP}+\mathrm{GPC} 3$ & $0.789(0.723-0.845)$ & $<0.0001$ & 84.07 & 61.33 & 76.6 & 71.9 \\
\hline $\mathrm{AFP}+\mathrm{GP73}$ & $0.749(0.680-0.809)$ & $<0.0001$ & 49.6 & 92.0 & 90.3 & 54.8 \\
\hline$A F P+D C P$ & $0.653(0.580-0.72 I)$ & $<0.0001$ & 37.2 & 94.7 & 91.3 & 50.0 \\
\hline GPC3+GP73 & $0.733(0.664-0.795)$ & $<0.0001$ & 65.5 & 76.0 & 80.0 & 58.2 \\
\hline GPC3+DCP & $0.695(0.624-0.760)$ & $<0.0001$ & 62.8 & 76.0 & 79.8 & 57.6 \\
\hline$A F P+G P C 3+G P 73$ & $0.802(0.738-0.856)$ & $<0.0001$ & 63.7 & 82.7 & 84.7 & 60.2 \\
\hline$A F P+G P C 3+D C P$ & $0.777(0.710-0.834)$ & $<0.0001$ & 63.7 & 81.3 & 83.7 & 59.8 \\
\hline GPC3+GP73+DCP & $0.720(0.650-0.783)$ & $<0.0001$ & 64.6 & 76.0 & 80.2 & 58.8 \\
\hline $\mathrm{AFP}+\mathrm{GPC} 3+\mathrm{GP} 73+\mathrm{DCP}$ & $0.790(0.724-0.846)$ & $<0.0001$ & 62.8 & 84.0 & 85.5 & 60.0 \\
\hline
\end{tabular}

Note: AUC value of biomarkers $<0.6$ is not presented.

Abbreviations: AFP, $\alpha$-fetoprotein; GPC3, glypican 3; GP73, golgi protein 73; DCP, des- $\gamma$-carboxy prothrombin; AUC, area under curve; Sen, sensitivity; Sep, specificity; PPV, positive predictive value; NPV, negative predictive value; HCC, hepatocellular carcinoma; LC, liver cirrhosis; CHB, chronic hepatitis B virus infection; HC, healthy controls.

AFP is the most widely utilized serologic marker for HCC, and our data support its diagnostic advantage over the other three markers. However, the diagnostic value of the other three markers in this study was slightly different from what has been previously reported. Jorge A. Marrero et al reported that DCP had a better sensitivity $(89 \%$; $95 \%$ CI, 77\%-95\%) and specificity (95\%; 95\% CI, 82\%-96\%) than AFP for differentiating patients with $\mathrm{HCC}$ from those with cirrhosis and CHB ${ }^{20}$ However, some scholars have suggested that DCP is not effective in the early diagnosis of liver cancer, ${ }^{21}$ and that AFP has an absolute advantage over DCP in the diagnosis of early or very early liver cancer, ${ }^{22}$ and this advantage is more obvious in the early diagnosis of $\mathrm{HCC}^{23}$ Diagnosis using a combination of 
Table 4 The Value of Serum AFP, GPC3, GP73 and DCP in the Very Early Diagnosis of HCC

\begin{tabular}{|c|c|c|c|c|c|c|}
\hline Biomarkers & AUC $(95 \% \mathrm{Cl})$ & $P$ value & Sen (\%) & Spe (\%) & PPV (\%) & NPV (\%) \\
\hline \multicolumn{7}{|l|}{ vs $\mathrm{LC}, \mathrm{CHB}, \mathrm{HC}$} \\
\hline AFP & $0.726(0.662-0.784)$ & $<0.0001$ & 65.0 & 76.7 & 38.2 & 90.8 \\
\hline GPC3 & $0.601(0.533-0.666)$ & 0.017 & 35.0 & 85.0 & 34.1 & 85.5 \\
\hline $\mathrm{AFP}+\mathrm{GPC} 3$ & $0.75 I(0.688-0.807)$ & $<0.0001$ & 75.0 & 71.7 & 37.0 & 92.8 \\
\hline $\mathrm{AFP}+\mathrm{GP73}$ & $0.725(0.66 I-0.783)$ & $<0.0001$ & 65.0 & 76.7 & 38.2 & 90.8 \\
\hline $\mathrm{AFP}+\mathrm{DCP}$ & $0.742(0.679-0.798)$ & $<0.0001$ & 65.0 & 76.1 & 37.7 & 90.7 \\
\hline GPC3+GP73 & $0.601(0.533-0.666)$ & 0.0177 & 35.0 & 85.0 & 34.1 & 85.5 \\
\hline $\mathrm{AFP}+\mathrm{GPC} 3+\mathrm{GP73}$ & $0.750(0.688-0.806)$ & $<0.0001$ & 75.0 & 71.7 & 33.3 & 89.7 \\
\hline $\mathrm{AFP}+\mathrm{GPC} 3+\mathrm{DCP}$ & $0.752(0.689-0.807)$ & $<0.0001$ & 75.0 & 71.7 & 37.0 & 92.8 \\
\hline $\mathrm{AFP}+\mathrm{GPC} 3+\mathrm{GP73}+\mathrm{DCP}$ & $0.750(0.687-0.806)$ & $<0.0001$ & 75.0 & 71.7 & 37.0 & 92.8 \\
\hline \multicolumn{7}{|l|}{ vs LC,CHB } \\
\hline AFP & $0.688(0.613-0.757)$ & 0.0002 & 42.5 & 93.8 & 68.0 & 84.1 \\
\hline $\mathrm{AFP}+\mathrm{GPC} 3$ & $0.703(0.629-0.77 I)$ & $<0.0001$ & 42.5 & 96.2 & 76.2 & 83.9 \\
\hline $\mathrm{AFP}+\mathrm{GP73}$ & $0.687(0.61 \mathrm{I}-0.756)$ & $<0.0001$ & 42.5 & 93.8 & 68.0 & 84.1 \\
\hline $\mathrm{AFP}+\mathrm{DCP}$ & $0.700(0.626-0.768)$ & 0.0001 & 42.5 & 93.8 & 68.0 & 84.1 \\
\hline $\mathrm{AFP}+\mathrm{GPC} 3+\mathrm{GP73}$ & $0.702(0.627-0.770)$ & $<0.0001$ & 42.5 & 93.8 & 68.0 & 84.1 \\
\hline $\mathrm{AFP}+\mathrm{GPC} 3+\mathrm{DCP}$ & $0.704(0.629-0.77 I)$ & $<0.0001$ & 42.5 & 96.2 & 77.3 & 84.5 \\
\hline $\mathrm{AFP}+\mathrm{GPC} 3+\mathrm{GP} 73+\mathrm{DCP}$ & $0.702(0.627-0.770)$ & $<0.0001$ & 42.5 & 94.6 & 70.8 & 84.2 \\
\hline \multicolumn{7}{|l|}{ vs LC } \\
\hline AFP & $0.704(0.612-0.785)$ & $<0.0001$ & 65.0 & 70.7 & 54.2 & 79.1 \\
\hline $\mathrm{AFP}+\mathrm{GPC} 3$ & $0.704(0.61 \mathrm{I}-0.785)$ & $<0.0001$ & 75.0 & 61.3 & 50.8 & 82.1 \\
\hline $\mathrm{AFP}+\mathrm{GP73}$ & $0.70 \mathrm{I}(0.609-0.783)$ & $<0.0001$ & 65.0 & 70.7 & 54.2 & 79.1 \\
\hline $\mathrm{AFP}+\mathrm{DCP}$ & $0.692(0.599-0.774)$ & $<0.001$ & 42.5 & 94.7 & 81.0 & 75.5 \\
\hline $\mathrm{AFP}+\mathrm{GPC} 3+\mathrm{GP73}$ & $0.702(0.610-0.784)$ & $<0.0001$ & 75.0 & 61.3 & 50.8 & 82.1 \\
\hline $\mathrm{AFP}+\mathrm{GPC} 3+\mathrm{DCP}$ & $0.708(0.616-0.789)$ & $<0.0001$ & 75.0 & 62.7 & 51.7 & 82.5 \\
\hline $\mathrm{AFP}+\mathrm{GPC} 3+\mathrm{GP} 73+\mathrm{DCP}$ & $0.703(0.6 \mathrm{II}-0.785)$ & $<0.0001$ & 42.5 & 94.7 & 81.0 & 75.5 \\
\hline
\end{tabular}

Note: AUC value of biomarkers $<0.6$ is not presented.

Abbreviations: AFP, $\alpha$-fetoprotein; GPC3, glypican 3; GP73, golgi protein 73; DCP, des- $\gamma$-carboxy prothrombin; AUC, area under curve; Sen, sensitivity; Sep, specificity; PPV, positive predictive value; NPV, negative predictive value; HCC, hepatocellular carcinoma; LC, liver cirrhosis; CHB, chronic hepatitis B virus infection; HC, healthy controls.

these two markers has been proposed; although neither AFP nor DCP is good at early diagnosis, their combination showed a significantly improved diagnosis and treatment effect, $^{24}$ similar to our conclusion. In addition to increasing the utility for early diagnosis, the combination of the two markers showed an improvement in the diagnostic efficacy, ${ }^{25}$ which may be related to the sensitivity of DCP. $^{26}$ For GPC3, a specific increase in mRNA levels in liver cancer tissue leads to an increase in protein levels. Therefore, clinically, many clinical tools, such as tissue biopsy $^{27}$ or immunohistochemistry, ${ }^{28}$ are available. However, there is a current trend in serological testing. Early reports show that AFP combined with GPC3 can significantly increase the detection rate of early $\mathrm{HCC},{ }^{14}$ consistent with our findings. Finally, for GP73, our findings differ greatly from those previously published. Our data showed that serum GP73 levels were not high, and its AUC value was less than 0.6 in all the sub-groups, indicating limited power to discriminate patients with and without liver cancer. However, in 2005, Jorge A. Marrero et al reported that GP73 was significantly better than AFP in the early diagnosis of liver cancer, with an AUC value of 0.79 vs $0.61{ }^{19}$ Moreover, GP73 was significantly superior to AFP in detecting the postoperative recurrence of liver cancer. ${ }^{15}$ We speculate that these differences may be mainly caused by the following factors: one, the subjects included in the different studies were from different countries, with ethnic and geographical differences, which will have a certain impact on the results; and two, our study included a single HBV-related non-HCC control group (including CHB and HBV-related LC) and the HCC groups, while other studies did not have clearly refined groups. The non-cancer group may have included patients with various types of hepatitis and cirrhosis caused by different factors, such as alcohol, drugs and bile damage, etc., and the cancer group may have 
A

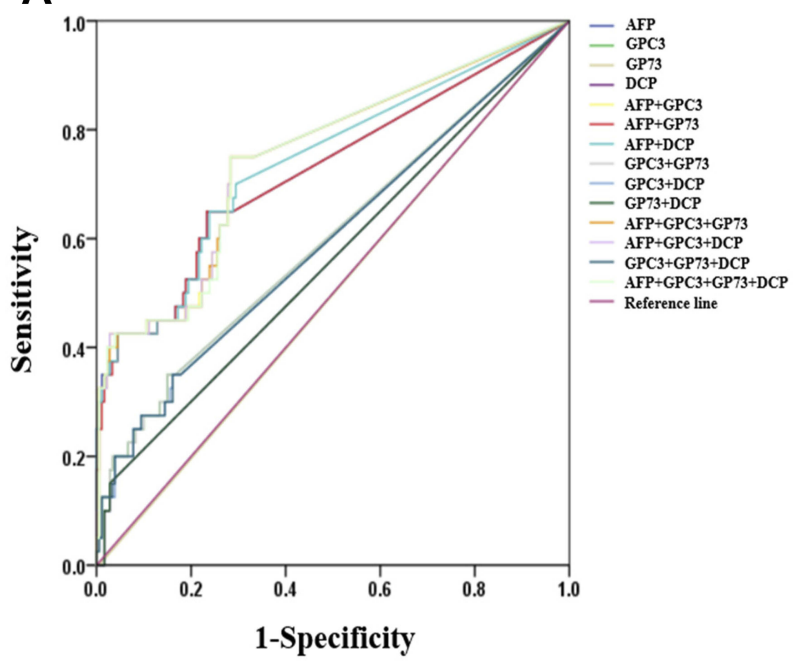

B

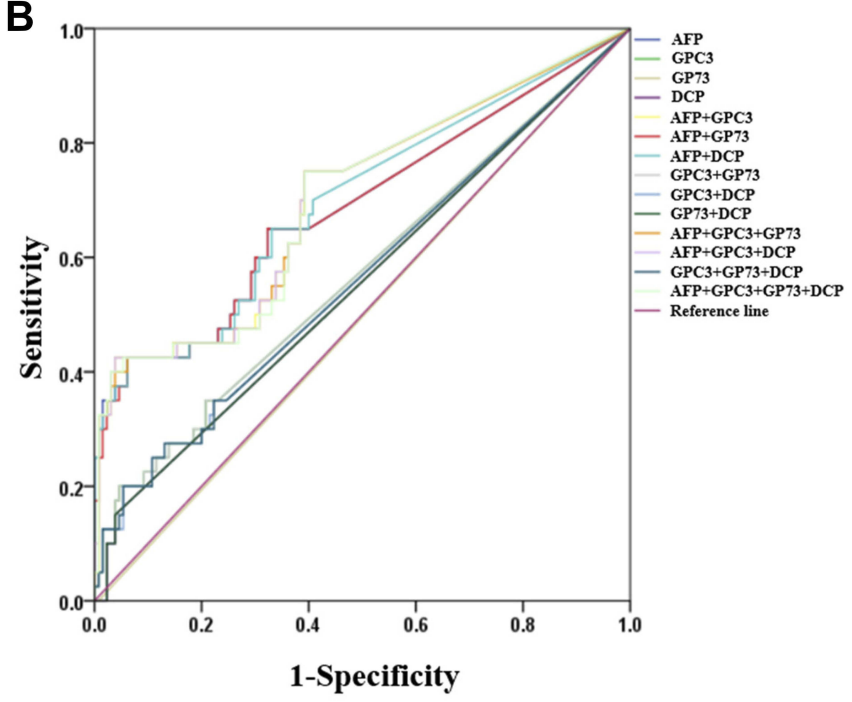

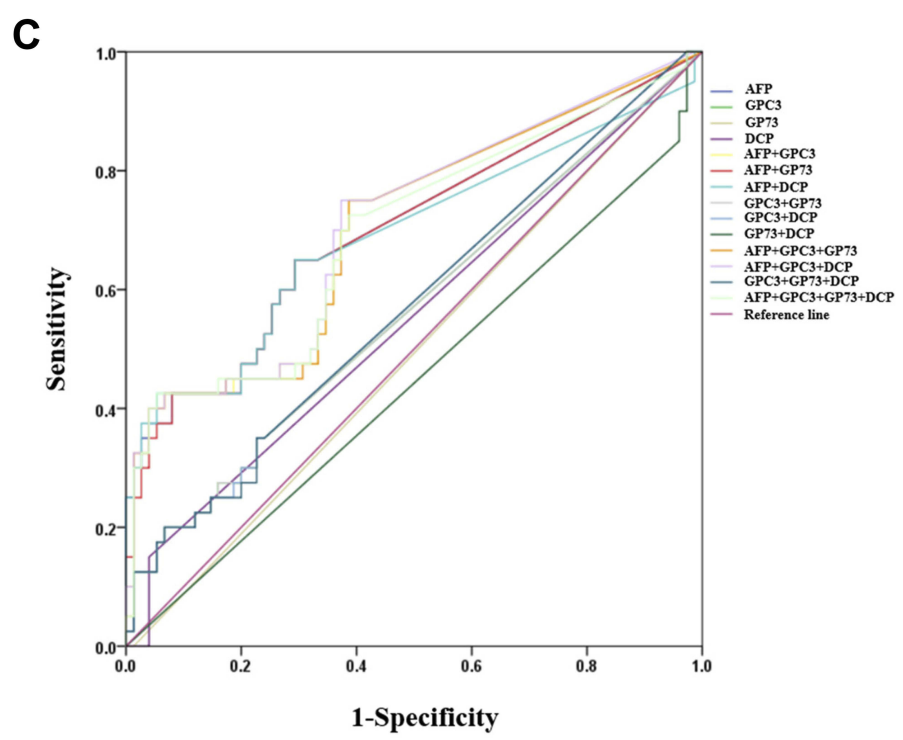

Figure 3 The value of serum AFP, GPC3, GP73 and DCP in the very early diagnosis of HBV-related HCC. (A) Very early stage HCC vs LC, CHB, HC. (B) Very early stage vs LC, CHB. (C) Very early stage vs LC.

Abbreviations: HCC, hepatocellular carcinoma; LC, liver cirrhosis; CHB, chronic hepatitis B virus infection; HC, healthy controls; AFP, $\alpha$-fetoprotein; GPC3, glypican 3; GP73, golgi protein 73; DCP, des- $\gamma$-carboxy prothrombin.

included various mixed types of HCC, leading to major differences in inclusions and exclusion criteria.

Liquid biopsy has considerable advantages over tissue biopsy, it is easy to perform and avoids the issue of tumour heterogeneity tissue samples making it a good source of tumor markers. Currently, the commonly used markers are nucleic acids such as non-coding RNA (lncRNA and miRNA) and cfDNA, proteins and circulating tumour cells (CTCs). Nucleic acid markers such as non-coding RNA and cfDNA are highly sensitive and can be detected at a very early stage in the development of liver cancer, ${ }^{29-34}$ but these markers have certain drawbacks. Non-coding
RNAs single-stranded and do not have sufficient stability. cfDNA originates from not only tumuor cells, but also many ageing cells and inflammatory cells. Thus, specificity is affected to some extent. Therefore, nucleic acid markers are now largely developed towards based on their biology, such as methylation ${ }^{35,36}$ and mutation, ${ }^{37-39}$ to provide insight into treatment choice or potential drug resistance. As complete cells, CTCs can provide all the same information as nucleic acids. Tumuor cells that enter the blood are more malignant than primary tumour cells. Research on CTCs has focused more on targeted anticancer therapy. However, due to the characteristics of intact cells, 
a certain degree of malignancy must be reached before primary tumuor will enter the blood, which limits the application of CTCs in the early diagnosis of liver cancer. At present, the application of CTCs in liver cancer is basically limited to staging, prognosis ${ }^{40}$ and therapeutic response evaluation. ${ }^{41-44}$ Protein markers are classic tumuor markers with known stability. In general, good protein markers have considerable advantages in sensitivity and specificity. In the future, we can combine these various markers to better monitor the occurrence and development of liver cancer. (To reviewer 1)

Our results are very encouraging but there are several important limitations of our study. We divided the noncancer groups and cancer groups into many sub-categories. Although the total number of enrolled patients was appropriate, the average number of participants in each subgroup was relatively small. In addition, all the included subjects were Chinese; this study was conducted at singlecenter with participants of a single ethnicity.

\section{Conclusions}

In conclusion, our data show that as an individual marker, AFP has an advantage over the other three serum markers in the very early and early diagnosis of HBV-related HCC. When AFP was combined with one of the other three markers (DCP, GPC3 and GP73), the AUC, sensitivity and specificity could be increased. AFP remained the best marker for the very early diagnosis of HBV-related HCC, and the addition of one or more markers did not significantly improve the diagnostic accuracy. Additional studies are needed to determine whether the same diagnostic method can be applied to all ethnic groups and all underlying aetiologies of liver disease; this information would have great clinical significance.

\section{Abbreviations}

AFP, alpha-fetoprotein; DCP, des-gamma-carboxyprothrombin; GPC3, gypican-3; GP73, Golgi protein 73; HCC, hepatocellular carcinoma; $\mathrm{HBV}$, hepatitis $\mathrm{B}$ virus; $\mathrm{CHB}$, chronical HBV infection; LC, liver cirrhosis; ROC, receiver operating characteristics; AUC, area under curve; TBil, total bilirubin; ALT, alanine aminotransferase; AST, aspartate aminotransferase; TP, total protein.

\section{Data Sharing Statement}

The datasets used in the current study are available from the corresponding author on reasonable request.

\section{Ethics Approval and Consent to Participate}

Informed consent was obtained from each patient prior to study enrolment. The study was approved by the ethics review board of the institutional ethics committee of Beijing YouAn Hospital.

\section{Funding}

This study was financially supported by Beijing Municipal Science \& Technology Commission (D171100003117004). The funders had no role in study design, data collection and analysis, decision to publish, or manuscript preparation.

\section{Disclosure}

The authors report no conflicts of interest in this work.

\section{References}

1. Zheng R, Qu C, Zhang S, et al. Liver cancer incidence and mortality in China: temporal trends and projections to 2030. J Cancer Res. 2018;30(6):571-579.

2. Wanqing Chen.Cancer statistics:updated cancer burden in China. Chinese J Cancerr Res. 2015;27(1):1.

3. Freddie B, Jacques F, Isabelle S, Siegel RL, Torre LA, Jemal A, Clinicians JAJCACJf. Global cancer statistics 2018: GLOBOCAN estimates of incidence and mortality worldwide for 36 cancers in 185 countries. CA: Cancer J Clin. 2015.

4. Siegel RL, Miller KD, Jemal A. Cancer statistics. CA Cancer J Clin. 2018;60(5):277-300.

5. Chen CJ, Liang K-Y, Chang A-S, et al. Effects of hepatitis B virus, alcohol drinking, cigarette smoking and familial tendency on hepatocellular carcinoma. Hepatology. 1991;13(3):398-406.

6. Dienstag JLJNEJoM. Hepatitis B virus infection. New England J Med. 2008;359(14):1486.

7. Benhenda S, Cougot D, Neuveut C, Buendia MAJV. Liver cell transformation in chronic HBV infection. Viruses. 2009;1 (3):630-646. doi:10.3390/v1030630

8. Ohkura Y, Sasaki K, Matsuda M, Hashimoto M, Watanabe GJBS. Long-term prognosis after resection of cryptogenic hepatocellular carcinoma. BMC surgery. 2015;15(1):115.

9. Ryder SJGMJ. British society of gastroenterology. Guidelines for the diagnosis and treatment of hepatocellular carcinoma (HCC) in adults. Gut. 2004;52(52 Suppl 3):iii1.

10. Ebara M, Ohto M, Kondo F. Strategy for early diagnosis of hepatocellular carcinoma (HCC). Ann Acad Med Singapore. 1989;18(1):83.

11. Hennedige T, Venkatesh SK. Imaging of hepatocellular carcinoma: diagnosis, staging and treatment monitoring. Cancer Imaging. 2013;12(3):530-547. doi:10.1102/1470-7330.2012.0044

12. Beale G, Chattopadhyay D, Gray J, et al. AFP, PIVKAII, GP3, SCCA-1 and follisatin as surveillance biomarkers for hepatocellular cancer in non-alcoholic and alcoholic fatty liver disease. $B M C$ Cancer. 2008;8(1):200.

13. Ji J, Wang H, Li Y, et al. Diagnostic evaluation of des-gammacarboxy prothrombin versus $\alpha$-fetoprotein for hepatitis B virusrelated hepatocellular carcinoma in China: a large-scale, multicentre study. PloS one. 2016;11(4):e0153227.

14. Capurro M, Wanless IR, Sherman M, et al. Glypican-3: a novel serum and histochemical marker for hepatocellular carcinoma. Gastroenterology. 2003;125(1):89-97. doi:10.1016/s0016-5085(03) 00689-9 
15. Mao Y, Yang $\mathrm{H}$, Xu H, et al. Golgi protein 73 (GOLPH2) is a valuable serum marker for hepatocellular carcinoma. Gut. 2010;59(12):1687-1693. doi:10.1136/gut.2010.214916

16. Seo SI, Su SK, Bo YC, Sang HL, Hepato-gastroenterology MKJJ. Clinical significance of elevated serum Alipha-Fetoprotein (AFP) level in acute viral hepatitis A (AHA). Hepato-Gastroenterology. 2013;60(127):1592-1596.

17. LIEBMAN HJNEJoM. Desgamma-carboxy (abnormal) prothrombin as a serum marker of primary hepatocellular carcinoma. The New England Journal of Medicine. 1984;310(22):1427.

18. Yorita K, Takahashi N, Takai H, et al. Prognostic significance of circumferential cell surface immunoreactivity of glypican-3 in hepatocellular carcinoma. Liver Int. 2015;31(1):120-131.

19. Marrero JA, Romano PR, Nikolaeva O, et al. GP73, a resident Golgi glycoprotein, is a novel serum marker for hepatocellular carcinoma. J Hepatol. 2005;43(6):1007-1012. doi:10.1016/j.jhep.2005.05.028

20. Marrero JA, Su GL, Wei W, et al. Des-gamma carboxyprothrombin can differentiate hepatocellular carcinoma from nonmalignant chronic liver disease in american patients. Hepatology. 2003;37 (5):1114-1121. doi:10.1053/jhep.2003.50195

21. Mita Y, Aoyagi Y, Yanagi M, Suda T, Suzuki Y, Asakura HJC. The usefulness of determining des-gamma-carboxy prothrombin by sensitive enzyme immunoassay in the early diagnosis of patients with hepatocellular carcinoma. J Am Cancer Soc. 2015;82(9):1643-1648.

22. Marrero JA, Feng Z, Wang Y, et al. $\alpha$-fetoprotein, des- $\gamma$ carboxyprothrombin, and lectin-bound $\alpha$-fetoprotein in early hepatocellular carcinoma. Gastroenterology. 2009;137(1):110-118. doi:10.1053/j. gastro.2009.04.005

23. Chan CY, Lee S-D, Wu J-C, et al. The diagnostic value of the assay of des- $\gamma$-carboxy prothrombin in the detection of small hepatocellular carcinoma. Journal of hepatology. 1991;13(1):21-24.

24. Lok AS, Sterling RK, Everhart JE, et al. Des- $\gamma$-carboxy prothrombin and $\alpha$-fetoprotein as biomarkers for the early detection of hepatocellular carcinoma. Gastroenterology. 2010;138(2):493-502. doi:10. 1053/j.gastro.2009.10.031

25. Toyoda H, Kumada T, Tada $\mathrm{T}$, et al. Prognostic significance of a combination of pre- and post-treatment tumor markers for hepatocellular carcinoma curatively treated with hepatectomy. $J$ Hepatol. 2012;57(6):1251-1257. doi:10.1016/j.jhep.2012.07.018

26. Toyoda H, Kumada T, Kaneoka Y, et al. Prognostic value of pretreatment levels of tumor markers for hepatocellular carcinoma on survival after curative treatment of patients with HCC. J Hepatol. 2008;49 (2):223-232. doi:10.1016/j.jhep.2008.04.013

27. Tommaso LD, Destro A, Seok JY, et al. The application of markers (HSP70 GPC3 and GS) in liver biopsies is useful for detection of hepatocellular carcinoma. $J$ Hepatol. 2009;50(4):746-754. doi:10.1016/j.jhep.2008.11.014

28. Tremosini S, Forner A, Boix L, et al. Prospective validation of an immunohistochemical panel (glypican 3, heat shock protein 70 and glutamine synthetase) in liver biopsies for diagnosis of very early hepatocellular carcinoma. Gut. 2012;61(10):1481-1487. doi:10.1136/ gutjnl-2011-301862

29. Bao H, Su H. Long noncoding RNAs act as novel biomarkers for hepatocellular carcinoma: progress and prospects. BioMed Res Int. 2017;2017:6049480.

30. Ali HEA, Abdel Hameed R, Effat H, et al. Circulating microRNAs panel as a diagnostic tool for discrimination of HCV-associated hepatocellular carcinoma. Biomed Res Int. 2017;41(4):e51-e62.
31. Huang A, Zhang X, Zhou SL, et al. Plasma circulating cell-free DNA integrity as a promising biomarker for diagnosis and surveillance in patients with hepatocellular carcinoma. $J$ Cancer. 2016;7 (13):1798-1803. doi:10.7150/jca.15618

32. Huang $\mathrm{YH}$, Liang $\mathrm{KH}$, Chien RN, et al. A circulating MicroRNA signature capable of assessing the risk of hepatocellular carcinoma in cirrhotic patients. Sci Rep. 2017;7(1):523. doi:10.1038/s41598-01700631-9

33. Musaddaq G, Shahzad N, Ashraf MA. Circulating liver-specific microRNAs as noninvasive diagnostic biomarkers of hepatic diseases in human. Biomarkers. 2019;24(2):103-109. doi:10.1080/135475 0X.2018.1528631

34. Xiong Y, Xie CR, Zhang S, Chen J, Yin ZY. Detection of a novel panel of somatic mutations in plasma cell-free DNA and its diagnostic value in hepatocellular carcinoma. Gut. 2019;11:5745-5756.

35. Cai J, Chen L. Genome-wide mapping of 5-hydroxymethylcytosines in circulating cell-free DNA as a non-invasive approach for early detection of hepatocellular carcinoma. Clin Cancer Res. 2019;68 (12):2195-2205.

36. Hlady RA, Zhao X, Pan X, et al. Genome-wide discovery and validation of diagnostic DNA methylation-based biomarkers for hepatocellular cancer detection in circulating cell free DNA. Theranostics. 2019;9(24):7239-7250. doi:10.7150/thno.35573

37. Ikeda S, Tsigelny IF, Skjevik AA, et al. Next-generation sequencing of circulating tumor DNA reveals frequent alterations in advanced hepatocellular carcinoma. Oncologist. 2018;23(5):586-593. doi:10. 1634/theoncologist.2017-0479

38. Kaseb AO, Sanchez NS, Sen S, et al. Molecular profiling of hepatocellular carcinoma using circulating cell-free DNA. Clin Cancer Res. 2019;25(20):6107-6118. doi:10.1158/1078-0432.CCR-18-3341

39. Liebs S, Keilholz U, Kehler I, Schweiger C, Hayback J, Nonnenmacher A. Detection of mutations in circulating cell-free DNA in relation to disease stage in colorectal cancer. Cancer Med. 2019;8(8):3761-3769. doi:10.1002/cam4.2219

40. Schulze K, Gasch C, Staufer K, et al. Presence of EpCAM-positive circulating tumor cells as biomarker for systemic disease strongly correlates to survival in patients with hepatocellular carcinoma. Int J Cancer. 2013;133(9):2165-2171. doi:10.1002/ijc.v133.9

41. Guo W, Sun YF, Shen MN, et al. Circulating tumor cells with stem-like phenotypes for diagnosis, prognosis, and therapeutic response evaluation in hepatocellular carcinoma. Clin Cancer Res. 2018;24(9):2203-2213. doi:10.1158/1078-0432.CCR-17-1753

42. Wang L, Li Y, Xu J, et al. Quantified postsurgical small cell size CTCs and $\operatorname{EpCAM}(+)$ circulating tumor stem cells with cytogenetic abnormalities in hepatocellular carcinoma patients determine cancer relapse. Cancer Lett. 2018;412:99-107. doi:10.1016/j.canlet. 2017.10.004

43. Wang S, Zheng Y, Liu J, Huo F, Zhou J. Analysis of circulating tumor cells in patients with hepatocellular carcinoma recurrence following liver transplantation. J Investig Med. 2018;66(5):1-6. doi:10.1136/ jim-2017-000655

44. Wang Z, Luo L, Cheng Y, et al. Correlation between postoperative early recurrence of hepatocellular carcinoma and mesenchymal circulating tumor cells in peripheral blood. J Gastrointest Surg. 2018;22 (4):633-639. doi:10.1007/s11605-017-3619-3 


\section{Publish your work in this journal}

OncoTargets and Therapy is an international, peer-reviewed, open access journal focusing on the pathological basis of all cancers, potential targets for therapy and treatment protocols employed to improve the management of cancer patients. The journal also focuses on the impact of management programs and new therapeutic agents and protocols on patient perspectives such as quality of life, adherence and satisfaction. The manuscript management system is completely online and includes a very quick and fair peer-review system, which is all easy to use. Visit http://www.dovepress.com/ testimonials.php to read real quotes from published authors.

Submit your manuscript here: https://www.dovepress.com/oncotargets-and-therapy-journal 